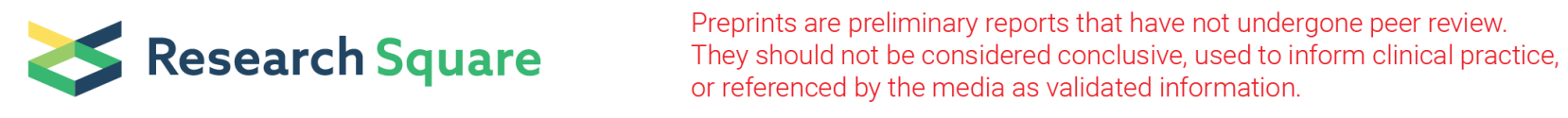

\title{
Development and Characterization of 135 SNP Markers in Chiton Acanthochitona Rubrolineatus
}

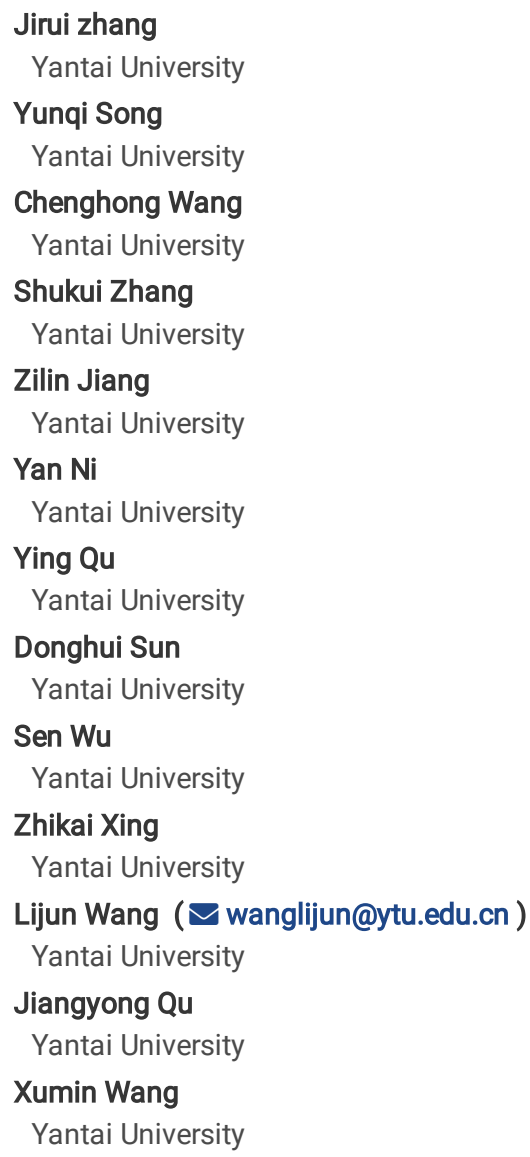

\section{Research Article}

Keywords: Acanthochitona rubrolineatus, SNPs markers, Population genetics, Diversity

Posted Date: August 17th, 2021

DOI: https://doi.org/10.21203/rs.3.rs-687709/v1

License: (c) (i) This work is licensed under a Creative Commons Attribution 4.0 International License. Read Full License

Version of Record: A version of this preprint was published at Conservation Genetics Resources on April 19th, 2022. See the published version at https://doi.org/10.1007/s12686-022-01264-5. 


\section{Abstract}

Acanthochitona rubrolineatus (Lischke, 1873) (Polyplacophora, Neoloricata, Cryptoplacidae) is an important species widely distributed in the middle and low tide zone of coastal intertidal zone along the China Sea coast. In recent years, the serious pollution of seawater has caused a sharp decline in the number of wild populations of $A$. rubrolineatus. Lacking of effective molecular markers limits the effective protection and management of this species. Studies for the isolation and characterization of $135 \mathrm{~A}$. rubrolineatus SNPs markers were carried out. The frequency of minor allele ranges from 0.0125 to 0.5000 . The observed heterozygosity and expected heterozygosity are $0.0000-0.9750,0.0731-0.6696$, respectively. The inbreeding value varies from -0.2902 to 0.9966 . Among them, there are 27 sites markablely differently from Hardy-Weinberg equilibrium $(P<0.05)$. The study of the polymorphic SNPs will provide a therotical basis for further analysis of population genetic analysis on $A$. rubrolineatus.

\section{Main Text}

Acanthochitona rubrolineatus belongs to Mollusca, Polyplacophora, which is a special branch in the evolution tree of Mollusca. In the 500 million years, their shape and living habits have not changed markly (Denny M W et al. 2008). Therefore, they have great value in studies of speciation and the evolution (Scherholz M et al. 2013). Now, studies on this species have been focused on its great economical values on new magnetic materials and biopharmaceuticals. According to ancient Chinese medical books, A. rubrolineatus can be used to treat leprosy and tuberculosis. Moreover, their extracts have immunological activity against tumor cells (Zhang $L$ et al. 2005). In addition, the radula teeth of $A$. rubrolineatus is one of the few structures that can synthesize nano-single-domain magnetic materials in vivo (Qian X. 2018; Fleissner G et al. 2007; Liu C. 2001). However, due to the frequent human activities and ecological environment destruction, the wild resources of $A$. rubrolineatus are declining sharply in China (Zhuang $S$ et al. 2001). To better protect wild resources, we performed an investigation in the population genetics of $A$. rubrolineatus.

Single nucleotide polymorphisms (SNPs), the third generation molecular marker, are mainly used to analyze DNA sequence polymorphism caused by genomic nucleotide level mutation (Kaikai et al. 2018). Genetic research based on SNP markers has several advantages, such as high frequency in genome and quickly genotype of many individuals (Schork $\mathrm{N}$ et al. 2000).

In this study, 40 A. rubrolineatus individuals were collected from Yantai coast, Shandong Province, China $\left(37^{\circ} 27^{\prime} \mathrm{N} / 121^{\circ} 30^{\prime} \mathrm{E}\right)$. Genomic DNA was extracted from blood samples using the DNeasy Blood \& Tissue kit (QIAGEN, Germany) according to the manufacturer's instructions. Then RAD library construction, sample indexing and pooling followed for the natural populations (Baird et al. 2008). 287581 putative SNPs were judged from $A$. rubrolineatus with Illumina HiSeq 4000 (Shanghai BIOZERON Co., Ltd) performing the paired end (150-bp) sequencing to obtain SNP markers resource.

Twenty-five primer pairs were designed by Primer v3.0. According to the instructions of manufacturer (GeneStar, Beijing, China), PCR in a $25 \mu$ l volume with GenStar PCR Mix. The PCR amplification cycle was as below: the initial denaturation at $94{ }^{\circ} \mathrm{C}$ was carried out for 5 min; then 40 cycles of $94{ }^{\circ} \mathrm{C}$ for $30{ }^{\circ} \mathrm{C}$, annealing for $30 \mathrm{~s}$ (for annealing temperatures of each primer pair, see Table 1 ), $72{ }^{\circ} \mathrm{C}$ for $30 \mathrm{~s}$; Finally, extendat $72{ }^{\circ} \mathrm{C}$ for 7 min. Amplified samples were purified by gelextraction and sequenced on ABI 3730 DNA analyzer (Applied Biosystems). For confirmed loci, the minor allele frequency (MAF), observed heterozygosity (HO), expected heterozygosity (HE), polymorphic information content (PIC), inbreeding coefficient (FIS), and P-values representing the deviations from the hardiy-weinberg equilibrium (HWEP) were involved in the statistics (Kalinowski et al. 2007).

135 SNP sequences were confirmed by Sanger sequencing. The minor allele frequency raged from 0.0125 to 0.5000 (Table 1 ). The observed heterozygosity and expected heterozygosity were $0.0000-0.9750$ and $0.0731-0.6696$, respectively. In addition, Polymorphism information content ranged from 0.0696 to 0.5870 , and inbreeding coefficient ranged from -0.2902 to 0.9966 . Only 27 loci showed significant deviations from HWE after Bonferroni correction $(\mathrm{P}<0.05)$. These SNP markers are of great significance to population genetic analysis of species.

\section{Declarations}

Acknowledgments: This work was supported by National Natural Science Foundation of China (31460562); Shandong Provincial Natural Science Foundation, China (ZR2020MD002); the Doctoral Science Research Foundation of Yantai University (SM15B01, SM19B70, SM19B28).

Conflicts of interest/Competing interests: The authors have no conflicts of interest to declare that are relevant to the content of this article.

Availability of data and material: All data analysed during this study are included in this published article.

Code availability: Not applicable.

Ethics approval: Not applicable.

Consent to participate: Not applicable.

Consent for publication: Not applicable.

Author contributions: Corresponding author contributed to the study conception and design. Shukui Zhang, Ying Qu and Zilin Jiang helped collect samples. Donghui Sun and Jirui Zhang were in charge of extracting genomic DNA. Zhikai Xing and Sen Wu helped collect data. Yan Ni, Chenghong 
Wang and Yunqi Song were in charge of compiling the data. The first draft of the manuscript was written by Jirui Zhang and all authors commented on previous versions of the manuscript. Corresponding author read and approved the final manuscript.

\section{References}

1. Denny MW, Gaines SD (2007) Encyclopedia of tidepools and rocky shores. Zool J Linn Soc-Lond. 157,753. https://doi.org10.1111/j.10963642.2008.00520.x

2. Scherholz M, Redl E, Wollesen T, Todt C, Wanninger A (2013) Aplacophoran mollusks evolved from ancestors with polyplacophoran-like features. Curr Biol: CB, 23 (21), 2103-2134. http://doi.org/10.1016/j.cub.2013.08.056

3. Zhang L, Xiao F, Han L (2005) Antitumor and immune regulation activities of the extracts of some Chinese marine invertebrates. Journal of marine Limnology of China 23 (1): 110-117

4. Qian X (2018) Magnetite in the radula teeth of chiton. Journal of Sichuan University (Natural Science Edition) $55,788$. http://doi.org/10.3969/j.issn.0490-6756.2018.04.022

5. Fleissner G, Stahl B, Thalau P, Fleissner G (2007) A novel concept of fe-mineral-based magnetoreception: histological and physicochemical data from the upper beak of homing pigeons. Sci Nat-Heidelberg, 94 (8), 631-642. http://doi.org/10.1007/s00114-007-0236-0

6. Liu C (2001) Morpholopy and mineral contents of the radula of chiton (Acanthochiton rubrolineatus lischke) Acta Zool. Sin (5),553-557. http://doi.org/CNKI:SUN:BEAR.0.2001-05-012

7. Zhuang S, Wang K, Chen L (2001) Study on Invertebrate Communities in Rocky Intertidal Zones Infuenced by Human Activities. J. Oceanogr. Huanghai Bohai Seas 19, 54-64. http://doi.org/10.3969/j.issn.1671-6647.2001.03.007

8. Kaikai L, Hong Y, Qi L (2018) Development and characterization of 108 SNP markers in the Iwagakioyster, Crassostrea nippona. Conserv Genet Resour 11 (4) :437-442. http://doi.org/10.1007/s12686-018-1047-7

9. Nicholas J Schork, Daniele Fallin , Jerry S Lanchbury (2000) Single nucleotide polymorphisms and the future of genetic epidemiology. Clin Genet 58:250-264. http://doi.org/10.1034/j.1399-0004.2000.580402.x

10. Baird NA, Etter PD, Atwood TS, Currey MC, Shiver AL, Lewis ZA, Selker EU, Cresko WA, Johnson EA (2008) Rapid SNP discovery and genetic mapping using sequenced RAD markers. Plos ONE 3: e63376. https://doi.org/10.1371/journal.pone.0003376

11. Kalinowski ST, Taper ML, Marshall TC (2007) Revising how the computer program CERVUS accommodates genotyping error increases success in paternity assignment. Mol Ecol 16:1099-11

\section{Tables}

Table 1 Characterization of 135 SNP markers in chiton Acanthochitona rubrolineatus. 
Primer sequence $\left(5^{\prime}-3^{\prime}\right)$

Size Tm SNP SNP

HWEP PIC MAF

\begin{tabular}{|c|c|c|c|c|c|c|c|c|c|c|c|}
\hline $\begin{array}{l}\text { Primer } \\
\text { ID }\end{array}$ & Primer sequence $\left(5^{\prime}-3^{\prime}\right)$ & $\begin{array}{l}\text { Size } \\
\text { (bp) }\end{array}$ & $\begin{array}{l}\mathrm{Tm} \\
\left({ }^{\circ} \mathrm{C}\right)\end{array}$ & $\begin{array}{l}\text { SNP } \\
\text { type }\end{array}$ & $\begin{array}{l}\text { SNP } \\
\text { position }\end{array}$ & & & & HWEP & $P I C$ & MAF \\
\hline \multirow[t]{2}{*}{110} & F: TCTGAGACACTGTGTGATTAAGAGA & 313 & 59 & $A / G$ & 67 & 0.9000 & 0.5013 & -0.2902 & 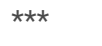 & 0.3725 & 0.4500 \\
\hline & R: TGATCATACGAGGAGGAAACGT & & & & & & & & & & \\
\hline \multirow[t]{2}{*}{115} & F: GAGAGAATGGGGGCGTCTTC & 317 & 60 & $\mathrm{G} / \mathrm{A}$ & 54 & 0.1750 & 0.2066 & 0.0680 & NS & 0.1910 & 0.0875 \\
\hline & R: CCTGTTACTGCAGTGCGTTG & & & & & & & & & & \\
\hline \multirow[t]{2}{*}{152} & F: GCAATGCCTGACTGTTGCTC & 338 & 60 & $\mathrm{~T} / \mathrm{A}$ & 52 & 0.3000 & 0.4418 & 0.1641 & NS & 0.3955 & 0.1500 \\
\hline & R: CGGGGAGAGGACGAAATCAG & & & & & & & & & & \\
\hline \multirow[t]{2}{*}{153} & F: ATGCCTGACTGTTGCTCACA & 262 & 60 & $T / G$ & 87 & 0.3000 & 0.4418 & 0.1641 & NS & 0.3955 & 0.1500 \\
\hline & R: TGTGGAGTATGTGTCTGGGC & & & & & & & & & & \\
\hline \multirow[t]{2}{*}{255} & F: TGCGCACTTAAATGACGAGC & 338 & 60 & $\mathrm{~T} / \mathrm{A}$ & 75 & 0.9000 & 0.5051 & -0.2868 & $\star \star \star *$ & 0.3744 & 0.4750 \\
\hline & R: ACGGGCCGTTAAATGCTAAC & & & & & & & & & & \\
\hline \multirow[t]{2}{*}{843} & F: CGACCGCCATTTCAGCTGTA & 296 & 61 & $\mathrm{~A} / \mathrm{C}$ & 85 & 0.8750 & 0.4984 & -0.2799 & $\star \star \star *$ & 0.3711 & 0.4375 \\
\hline & R: AAGTCAAAATTTCGCCCGGC & & & & & & & & & & \\
\hline \multirow[t]{2}{*}{1091} & F: AAGATGGCTGTAATTAACAAAAGA & 269 & 60 & $A / G$ & 108 & 0.7000 & 0.4608 & -0.2120 & NS & 0.3515 & 0.3500 \\
\hline & R: ACTCCAAAAGTACTGGGCCG & & & & & & & & & & \\
\hline \multirow[t]{2}{*}{1092} & F: AAGATGGCTGTAATTAACAAAAGA & 293 & 60 & $\mathrm{~A} / \mathrm{T}$ & 109 & 0.7000 & 0.4608 & -0.2120 & NS & 0.3515 & 0.3500 \\
\hline & R: ACTCCAAAAGTACTGGGCCG & & & & & & & & & & \\
\hline \multirow[t]{2}{*}{1145} & F: CAAGACGTGGCCTGAATCCT & 273 & 60 & $A / G$ & 95 & 0.5750 & 0.4149 & -0.1676 & NS & 0.3258 & 0.2875 \\
\hline & R: GCTGCCGAATATACAACCGC & & & & & & & & & & \\
\hline \multirow[t]{2}{*}{14760} & F: TCGCCAGTTGAGTATGACCC & 311 & 60 & $\mathrm{C} / \mathrm{T}$ & 53 & 0.9500 & 0.5051 & -0.3144 & 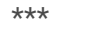 & 0.3744 & 0.4750 \\
\hline & R: ATGGCTGCCGTACTCAAGAG & & & & & & & & & & \\
\hline \multirow[t]{2}{*}{14955} & F: TGACTGGTCATTGTCCAAGGA & 319 & 60 & $\mathrm{C} / \mathrm{A}$ & 93 & 0.7250 & 0.4680 & -0.2213 & NS & 0.3554 & 0.3625 \\
\hline & R: ATTGTTGTGGAGGCGTTTGC & & & & & & & & & & \\
\hline \multirow[t]{2}{*}{15195} & F: TTCTACCCCTACGTGGACCA & 311 & 60 & $\mathrm{G} / \mathrm{C}$ & 68 & 0.9500 & 0.5051 & -0.3144 & 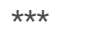 & 0.3744 & 0.4750 \\
\hline & R: ACCTCCGTATACGAGTCCTGA & & & & & & & & & & \\
\hline \multirow[t]{2}{*}{15433} & F: ATCTTACACTATTTATACACTGGGA & 337 & 59 & $\mathrm{C} / \mathrm{G}$ & 95 & 0.2750 & 0.2826 & -0.0030 & NS & 0.2516 & 0.1375 \\
\hline & R: AGAAGACAGATTCTGGAGCTGT & & & & & & & & & & \\
\hline \multirow[t]{2}{*}{15481} & F: TCATTGGTTAGCTGCTCTGGT & 336 & 60 & $\mathrm{~T} / \mathrm{C}$ & 66 & 0.8750 & 0.4984 & -0.2799 & 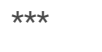 & 0.3711 & 0.4375 \\
\hline & R: TCATCGGTTTGCGTCAGTCA & & & & & & & & & & \\
\hline \multirow[t]{2}{*}{16111} & F: TGTCACTCAATCACACATTGTCT & 310 & 60 & $\mathrm{~T} / \mathrm{G}$ & 96 & 0.9750 & 0.5060 & -0.3221 & 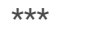 & 0.3748 & 0.4875 \\
\hline & R: TGGGGACAGAATAGTCTAGCCA & & & & & & & & & & \\
\hline \multirow[t]{2}{*}{16287} & F: CTACTGAGCTTCGGCCACAA & 334 & 60 & $A / G$ & 41 & 0.2000 & 0.1823 & -0.0462 & NS & 0.1638 & 0.1000 \\
\hline & R: GCGGCATTGTCAGAATAGGC & & & & & & & & & & \\
\hline \multirow[t]{2}{*}{16582} & F: TCAGAACTGCTTGACCCTGG & 273 & 60 & $A / G$ & 97 & 0.8000 & 0.4861 & -0.2499 & * & 0.3648 & 0.4000 \\
\hline & R: TAATGAAACGTGTGTCGCGC & & & & & & & & & & \\
\hline \multirow[t]{2}{*}{16714} & F: TGCTTGCGTTTAAACCACCG & 335 & 60 & $\mathrm{C} / \mathrm{T}$ & 73 & 0.9750 & 0.5060 & -0.3221 & $\star \star \star *$ & 0.3748 & 0.4875 \\
\hline & R: TGCTTGCGTTTAAACCACCG & & & & & & & & & & \\
\hline \multirow[t]{2}{*}{47003} & F: ACAAGAGGCTCCAAATTATCCTT & 278 & 60 & $\mathrm{~T} / \mathrm{C}$ & 77 & 0.8500 & 0.4949 & -0.2698 & ** & 0.3693 & 0.4250 \\
\hline & R: CCGTGGTTTGGCGGTAATTC & & & & & & & & & & \\
\hline \multirow[t]{2}{*}{63291} & F: GGGGCCCATAGCGTGAATT & 326 & 60 & $\mathrm{~T} / \mathrm{C}$ & 40 & 0.0750 & 0.3522 & 0.6463 & NS & 0.3080 & 0.0375 \\
\hline & R: TTCGACTGCTACACGGATCC & & & & & & & & & & \\
\hline
\end{tabular}




\begin{tabular}{|c|c|c|c|c|c|c|c|c|c|c|c|}
\hline \multirow[t]{2}{*}{63292} & F: GGGGCCCATAGCGTGAATT & 315 & 60 & $\mathrm{~T} / \mathrm{C}$ & 83 & 0.0750 & 0.3522 & 0.6463 & NS & 0.3080 & 0.0375 \\
\hline & R: GCCAGTCCGCCAGTTTAAGA & & & & & & & & & & \\
\hline \multirow[t]{2}{*}{64525} & F: GCTCCTGCTGGTTTTCGAAC & 318 & 60 & $\mathrm{G} / \mathrm{C}$ & 50 & 0.4750 & 0.4497 & -0.0453 & NS & 0.3652 & 0.2875 \\
\hline & R: TTCTGGTTCCCCTGCGTTTT & & & & & & & & & & \\
\hline \multirow[t]{2}{*}{65017} & F: GTAGGTTGCCAGCTCACTGT & 272 & 60 & $\mathrm{~A} / \mathrm{G}$ & 84 & 0.1250 & 0.3946 & 0.5068 & NS & 0.3557 & 0.1375 \\
\hline & R: GGAGCTAAGCACGCGTAGTA & & & & & & & & & & \\
\hline \multirow[t]{2}{*}{67289} & F: CGAACGATTAGAGCGGGACA & 260 & 60 & $\mathrm{C} / \mathrm{T}$ & 103 & 0.8500 & 0.4949 & -0.2698 & ** & 0.3693 & 0.4250 \\
\hline & R: GTAGGACCTAAACTGCCCGG & & & & & & & & & & \\
\hline \multirow[t]{2}{*}{67481} & F: TGTGTTTCCGTTGTTGTGGT & 325 & 60 & $\mathrm{~T} / \mathrm{C}$ & 88 & 0.8500 & 0.4949 & -0.2698 & ** & 0.3693 & 0.4250 \\
\hline & R: CTGGTGTGTGTATCTGCCCA & & & & & & & & & & \\
\hline \multirow[t]{2}{*}{67913} & F: GGGCAGGGGTGCAAAGTATA & 292 & 60 & $\mathrm{~A} / \mathrm{T}$ & 75 & 0.1750 & 0.5934 & 0.5352 & * & 0.5197 & 0.1875 \\
\hline & R: TCGCATCAACAACGTACACG & & & & & & & & & & \\
\hline \multirow[t]{2}{*}{68037} & F: TCCAGTTCTGCATGAAATTATCCA & 310 & 60 & $\mathrm{G} / \mathrm{A}$ & 97 & 0.2750 & 0.2402 & -0.0699 & NS & 0.2091 & 0.1375 \\
\hline & R: CGGGAACTCGGAAGAACCAA & & & & & & & & & & \\
\hline \multirow[t]{2}{*}{68108} & F: AGTTTGCAAGCAATTTCAAGA & 341 & 60 & $\mathrm{G} / \mathrm{A}$ & 41 & 0.9500 & 0.5051 & -0.3114 & $\star \star \star$ & 0.3744 & 0.4750 \\
\hline & R: ACGGAGGATTCGTTGCCATT & & & & & & & & & & \\
\hline \multirow[t]{2}{*}{97286} & F: GAATACCCTTCCCGTGCAGT & 324 & 60 & $\mathrm{G} / \mathrm{A}$ & 69 & 0.3250 & 0.3168 & -0.0300 & NS & 0.2771 & 0.1625 \\
\hline & R: GACTTTGCCGCCAAGCATAC & & & & & & & & & & \\
\hline \multirow[t]{2}{*}{97287} & F: GGAATACCCTTCCCGTGCAG & 281 & 60 & $\mathrm{G} / \mathrm{A}$ & 77 & 0.3250 & 0.3168 & -0.0300 & NS & 0.2771 & 0.1625 \\
\hline & R: CCAGGGGCTTGATATTGGCA & & & & & & & & & & \\
\hline \multirow[t]{2}{*}{97451} & F: TCCTCGGAACAACTAATCCT & 291 & 60 & $\mathrm{C} / \mathrm{T}$ & 35 & 0.9000 & 0.5013 & -0.2902 & $\star \star \star$ & 0.3725 & 0.4500 \\
\hline & R: GTCACCGCTGACTATCGACC & & & & & & & & & & \\
\hline \multirow[t]{2}{*}{97605} & F: CGAACGATTAGAGCGGGACA & 324 & 60 & $\mathrm{G} / \mathrm{A}$ & 95 & 0.3500 & 0.4722 & 0.1258 & NS & 0.3947 & 0.2750 \\
\hline & R: ATTTGACGGGACTCTGGTCG & & & & & & & & & & \\
\hline \multirow[t]{2}{*}{98716} & F: AGCGGTGAAACAGGTAAGGA & 338 & 61 & $\mathrm{~T} / \mathrm{C}$ & 43 & 0.9750 & 0.5060 & -0.3221 & $\star \star \star$ & 0.3748 & 0.4875 \\
\hline & R: CTCCCTCTGACTGGCTCCAT & & & & & & & & & & \\
\hline \multirow[t]{2}{*}{101130} & F: TCATGCCGAGTAGATATTTCCA & 306 & 59 & $\mathrm{G} / \mathrm{A}$ & 61 & 0.6250 & 0.5275 & -0.1133 & NS & 0.4278 & 0.3625 \\
\hline & R: AGAATCTGTACCGTTGCCTCT & & & & & & & & & & \\
\hline \multirow[t]{2}{*}{102257} & F: TGACTTGGTCAGGGACTTTGT & 292 & 60 & $\mathrm{G} / \mathrm{A}$ & 70 & 0.2750 & 0.2402 & -0.0699 & NS & 0.2091 & 0.1375 \\
\hline & R: ATCCCTGGAGCTGATGTCCT & & & & & & & & & & \\
\hline \multirow[t]{2}{*}{103281} & F: TGTAGTTCTTGTGATATATTGCACA & 336 & 60 & $\mathrm{G} / \mathrm{C}$ & 83 & 0.3000 & 0.3241 & 0.0323 & NS & 0.2688 & 0.2000 \\
\hline & R: GGCCAACGAATGTTTGGGAC & & & & & & & & & & \\
\hline \multirow[t]{2}{*}{105060} & F: GGCTTCGGCGATTTTCACG & 306 & 60 & $\mathrm{G} / \mathrm{A}$ & 106 & 0.4000 & 0.3532 & -0.0684 & NS & 0.2879 & 0.2250 \\
\hline & R: CCGTTCAGCGTTGACAGTTC & & & & & & & & & & \\
\hline \multirow[t]{2}{*}{115993} & F: CGTTTCCGTGTTGTGTGGAA & 330 & 59 & $\mathrm{C} / \mathrm{G}$ & 57 & 0.2250 & 0.3484 & 0.2012 & NS & 0.2996 & 0.1875 \\
\hline & R: AACGGCGTAAAACCCAATCA & & & & & & & & & & \\
\hline \multirow[t]{2}{*}{117340} & F: CCAGCCAATCAAATCTTGCGG & 371 & 60 & $\mathrm{G} / \mathrm{A}$ & 96 & 0.1500 & 0.1405 & -0.0307 & NS & 0.1291 & 0.0750 \\
\hline & R: CTGGAAAACTGCGCCGAATT & & & & & & & & & & \\
\hline \multirow[t]{2}{*}{117387} & F: TCCTGTGGAGCCATTGAAGT & 332 & 59 & $\mathrm{C} / \mathrm{T}$ & 31 & 0.8250 & 0.4908 & -0.2598 & ** & 0.3672 & 0.4125 \\
\hline & R: GCCCATGGACATCAATGATGG & & & & & & & & & & \\
\hline \multirow[t]{2}{*}{133437} & F: TTTGCTATCCGAGACGCACC & 294 & 60 & $\mathrm{C} / \mathrm{A}$ & 48 & 0.0750 & 0.0731 & -0.0102 & NS & 0.0696 & 0.0375 \\
\hline & R: CCTGAGGTTGAGGCAAGAGG & & & & & & & & & & \\
\hline 133496 & F: TTTTTCTGTAGAGAGAGATTTCCA & 326 & 56 & $\mathrm{~T} / \mathrm{C}$ & 89 & 0.2500 & 0.3911 & 0.2060 & NS & 0.3285 & 0.2250 \\
\hline & & & & age & & & & & & & \\
\hline
\end{tabular}


R: AGACAAAAGCTTCACAAAGGA

\begin{tabular}{|c|c|c|c|c|c|c|c|c|c|c|c|}
\hline \multirow[t]{2}{*}{153242} & F: AGCAAGAGAAACACAGCAGC & \multirow[t]{2}{*}{328} & \multirow[t]{2}{*}{60} & \multirow[t]{2}{*}{$\mathrm{G} / \mathrm{A}$} & \multirow[t]{2}{*}{97} & \multirow[t]{2}{*}{0.9750} & \multirow[t]{2}{*}{0.5060} & \multirow[t]{2}{*}{-0.3221} & \multirow[t]{2}{*}{$\star \star \star$} & \multirow[t]{2}{*}{0.3748} & \multirow[t]{2}{*}{0.4875} \\
\hline & R: CGCTGTTGTGTTTCTCTGGC & & & & & & & & & & \\
\hline \multirow[t]{2}{*}{159368} & F: CACCGGTCAAGGACCTGTC & 342 & 60 & $\mathrm{G} / \mathrm{T}$ & 97 & 0.9750 & 0.5060 & -0.3221 & $\star \star \star$ & 0.3748 & 0.4875 \\
\hline & R: AGGACGGAGGGACCACTAAA & & & & & & & & & & \\
\hline \multirow[t]{2}{*}{159555} & F: ATTGAATGAAGACTGGCGGT & 304 & 61 & $\mathrm{G} / \mathrm{A}$ & 71 & 0.8750 & 0.5060 & -0.2730 & $\star \star$ & 0.3748 & 0.4875 \\
\hline & R: AACAGCAACTAAGACCCCGC & & & & & & & & & & \\
\hline \multirow[t]{2}{*}{167472} & F: AATTCGAGTTGCCGGACTGT & 302 & 60 & $\mathrm{~T} / \mathrm{C}$ & 58 & 0.2750 & 0.3168 & 0.0545 & NS & 0.2771 & 0.1625 \\
\hline & R: GGTTTCCATCAGGTACCCCG & & & & & & & & & & \\
\hline \multirow[t]{2}{*}{167473} & F: AATTCGAGTTGCCGGACTGT & 358 & 60 & $\mathrm{C} / \mathrm{T}$ & 63 & 0.2750 & 0.3168 & 0.0545 & NS & 0.2771 & 0.1625 \\
\hline & R: ATACAGGAGTCGACGTCGGA & & & & & & & & & & \\
\hline \multirow[t]{2}{*}{170581} & F: АССTTCTCTGTGAACGATCCA & 302 & 60 & $A / G$ & 45 & 0.2250 & 0.2022 & -0.0545 & NS & 0.1797 & 0.1125 \\
\hline & R: ACCATCAGATCTGACACGGC & & & & & & & & & & \\
\hline \multirow[t]{2}{*}{186929} & F: CTCGACTACTGAGCTTCGGC & 342 & 60 & $\mathrm{~T} / \mathrm{C}$ & 60 & 0.4250 & 0.5389 & 0.0838 & NS & 0.4610 & 0.2875 \\
\hline & R: GCCAGATCTGCGTCTTTCCT & & & & & & & & & & \\
\hline \multirow[t]{2}{*}{186930} & F: GACTACTGAGCTTCGGCCAA & 341 & 60 & $\mathrm{G} / \mathrm{A}$ & 105 & 0.4250 & 0.5389 & 0.0838 & NS & 0.4610 & 0.2875 \\
\hline & R: CTCACGTGACGTCTGCAGAA & & & & & & & & & & \\
\hline \multirow[t]{2}{*}{188818} & F: TTCCGACACAGGTATGGACA & 340 & 60 & $A / G$ & 57 & 0.9750 & 0.5060 & -0.3221 & $\star \star \star ~$ & 0.3748 & 0.4875 \\
\hline & R: AGCCAAAACAGCATCCGGTA & & & & & & & & & & \\
\hline \multirow[t]{2}{*}{188819} & F: TTCCGACACAGGTATGGACA & 345 & 60 & $\mathrm{C} / \mathrm{T}$ & 69 & 0.9750 & 0.5060 & -0.3221 & $\star \star \star ~$ & 0.3748 & 0.4875 \\
\hline & R: TGTCAAATGGGTTGGTGCCT & & & & & & & & & & \\
\hline \multirow[t]{2}{*}{189292} & F: TTCAAGGCTGTGATTTTAGGGT & 353 & 60 & $\mathrm{~A} / \mathrm{T}$ & 92 & 0.7250 & 0.4908 & -0.1987 & NS & 0.3672 & 0.4125 \\
\hline & R: ACGCTCAAAGTACGAGTCCA & & & & & & & & & & \\
\hline \multirow[t]{2}{*}{189308} & F: ATTCCGTACTAGCCACATGT & 355 & 60 & $\mathrm{C} / \mathrm{G}$ & 89 & 0.9500 & 0.5051 & -0.3114 & 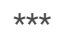 & 0.3744 & 0.4750 \\
\hline & R: AAAGACCAGCGAGTCATGGG & & & & & & & & & & \\
\hline 190334 & F: ATGCACTGATCCTGCTGGTT & 336 & 60 & $\mathrm{C} / \mathrm{A}$ & 64 & 0.1250 & 0.2085 & 0.2356 & NS & 0.1958 & 0.0625 \\
\hline & R: GCTGTTGGTTTCCGCACAAT & & & & & & & & & & \\
\hline 191667 & F: ATGGAAAGTGTGCGCTGGTA & 269 & 60 & $\mathrm{~T} / \mathrm{C}$ & 52 & 0.3250 & 0.5282 & 0.2087 & NS & 0.4613 & 0.2375 \\
\hline & R: AGTAGGCCACTGTGAAACGG & & & & & & & & & & \\
\hline 191668 & F: ATGGAAAGTGTGCGCTGGTA & 269 & 60 & $\mathrm{G} / \mathrm{C}$ & 102 & 0.3250 & 0.5282 & 0.2087 & NS & 0.4613 & 0.2375 \\
\hline & R: AGTAGGCCACTGTGAAACGG & & & & & & & & & & \\
\hline 192722 & F: AAAACGACCGGCACATGTTT & 349 & 60 & $\mathrm{C} / \mathrm{A}$ & 34 & 0.7000 & 0.6696 & -0.0693 & NS & 0.5870 & 0.3500 \\
\hline & R: ATCAATATGTGCCGGCCGTT & & & & & & & & & & \\
\hline 192723 & F: AAAACGACCGGCACATGTTT & 341 & 60 & $\mathrm{~T} / \mathrm{A}$ & 35 & 0.7000 & 0.6696 & -0.0693 & NS & 0.5870 & 0.3500 \\
\hline & R: ACACCTAGGCCCCATCAGAT & & & & & & & & & & \\
\hline 193182 & F: AGTTCCACGTACAATGCTAGAG & 311 & 61 & $\mathrm{C} / \mathrm{T}$ & 84 & 0.4750 & 0.5275 & 0.0265 & NS & 0.4278 & 0.3625 \\
\hline & R: ACGCTGTTTCCGGCACATAA & & & & & & & & & & \\
\hline 202668 & F: ATGATTCCACCTCGCTCACT & 274 & 59 & $\mathrm{~T} / \mathrm{C}$ & 86 & 0.0750 & 0.4839 & 0.7268 & NS & 0.4298 & 0.1375 \\
\hline & R: CGGCCATAAAACACCATGGT & & & & & & & & & & \\
\hline 216926 & F: GAACGATTAGAGCGGGGCAT & 322 & 60 & $\mathrm{~A} / \mathrm{T}$ & 78 & 0.1250 & 0.3225 & 0.4284 & NS & 0.2901 & 0.1375 \\
\hline & R: TGCAGCTCTTCCGATCTTCC & & & & & & & & & & \\
\hline 227919 & F: TCAGCACATTTTGGCGTTGG & 338 & 60 & $\mathrm{C} / \mathrm{T}$ & 96 & 0.1000 & 0.2924 & 0.4842 & NS & 0.2471 & 0.1750 \\
\hline & R: AAGTGGCCGCTTTAAGCAAC & & & & & & & & & & \\
\hline
\end{tabular}




\begin{tabular}{|c|c|c|c|c|c|c|c|c|c|c|c|}
\hline \multirow[t]{2}{*}{228342} & F: ATTGCACAATATAGTGACATCTATG & \multirow[t]{2}{*}{315} & \multirow[t]{2}{*}{58} & \multirow[t]{2}{*}{$\mathrm{G} / \mathrm{A}$} & \multirow[t]{2}{*}{66} & \multirow[t]{2}{*}{0.2750} & \multirow[t]{2}{*}{0.3085} & \multirow[t]{2}{*}{0.0512} & \multirow[t]{2}{*}{ NS } & \multirow[t]{2}{*}{0.2583} & \multirow[t]{2}{*}{0.1875} \\
\hline & R: AAGCGCACATCAATGAGACT & & & & & & & & & & \\
\hline \multirow[t]{2}{*}{228862} & F: TTCGGCAGTACAATCCCCAC & 336 & 60 & $\mathrm{C} / \mathrm{A}$ & 108 & 0.1750 & 0.3934 & 0.3720 & NS & 0.3530 & 0.0875 \\
\hline & R: TGAAAACAATGGCGGACGTG & & & & & & & & & & \\
\hline \multirow[t]{2}{*}{229977} & F: ACCTCACTGTTTCCTTCCGG & 268 & 60 & $\mathrm{~T} / \mathrm{C}$ & 108 & 0.4250 & 0.4617 & 0.0159 & NS & 0.3881 & 0.2625 \\
\hline & R: TAACGCAACTCAACTTCGCG & & & & & & & & & & \\
\hline \multirow[t]{2}{*}{236835} & F: TCTGAGACACTGTGTGATTAAGAGA & 299 & 60 & $\mathrm{C} / \mathrm{A}$ & 75 & 0.5000 & 0.3797 & -0.1423 & NS & 0.3047 & 0.2500 \\
\hline & R: ACGGTTGTAAGATGTTTGTGCC & & & & & & & & & & \\
\hline \multirow[t]{2}{*}{237216} & F: AGCATTGCCTGAGAACAGTCA & 323 & 60 & $\mathrm{G} / \mathrm{C}$ & 89 & 0.6000 & 0.4861 & -0.1111 & NS & 0.3648 & 0.4000 \\
\hline & R: AGTGGAAGGCAAGCATGTTG & & & & & & & & & & \\
\hline \multirow[t]{2}{*}{257954} & F: TCAAACTCGAAGTGTTCATTGA & 294 & 60 & $\mathrm{~A} / \mathrm{C}$ & 59 & 0.5000 & 0.5304 & 0.0120 & NS & 0.4103 & 0.4750 \\
\hline & R: AACTCATTCGCCCCTGTGAG & & & & & & & & & & \\
\hline \multirow[t]{2}{*}{258126} & F: TAAGAGGGCACGGAAGAGGA & 329 & 60 & $\mathrm{C} / \mathrm{G}$ & 97 & 0.9500 & 0.5063 & -0.3103 & $\star \star \star ~$ & 0.3750 & 0.5000 \\
\hline & R: ACGCATCTGCTTAGACAAATGC & & & & & & & & & & \\
\hline \multirow[t]{2}{*}{258935} & F: ACACCTGTAAACACGACTTGC & 270 & 60 & T/A & 47 & 0.5500 & 0.5215 & -0.0542 & NS & 0.4244 & 0.3500 \\
\hline & R: AGAAAGGCCGTATAGCTGCG & & & & & & & & & & \\
\hline \multirow[t]{2}{*}{268378} & F: TATCCGCTACCCCGCATAGA & 280 & 60 & $\mathrm{C} / \mathrm{T}$ & 65 & 0.4000 & 0.5051 & 0.0999 & NS & 0.3969 & 0.3750 \\
\hline & R: TGCGAAGTCTACCAGAACCG & & & & & & & & & & \\
\hline \multirow[t]{2}{*}{270177} & F: TAACTGCATTGTGTTGGGCT & 304 & 60 & $\mathrm{C} / \mathrm{T}$ & 106 & 0.2250 & 0.2022 & -0.0540 & NS & 0.1797 & 0.1125 \\
\hline & R: ACTGTTGATTACACCGGGCA & & & & & & & & & & \\
\hline \multirow[t]{2}{*}{270725} & F: CGCTTGACGTGACTTCTGGA & 263 & 60 & $\mathrm{C} / \mathrm{A}$ & 67 & 0.0750 & 0.1649 & 0.3572 & NS & 0.1553 & 0.0625 \\
\hline & R: GGCGTTTTGAGGCCTGTTATT & & & & & & & & & & \\
\hline 273467 & F: CAAAATGCAGCCGTATCTGGA & 343 & 60 & $\mathrm{~T} / \mathrm{C}$ & 73 & 0.1000 & 0.2684 & 0.4471 & NS & 0.2469 & 0.0500 \\
\hline & R: CGGTTCCAATCAGTGAAACACC & & & & & & & & & & \\
\hline 277151 & F: CTCAAGCCGTGGTGCTAAGA & 317 & 60 & $\mathrm{~T} / \mathrm{A}$ & 62 & 0.0250 & 0.3579 & 0.8633 & NS & 0.3205 & 0.0625 \\
\hline & R: CACACAAACGGAACCAACCA & & & & & & & & & & \\
\hline 286890 & F: TCCTAAACTACAAAATAATTCAATCCT & 344 & 60 & $\mathrm{~A} / \mathrm{T}$ & 50 & 0.4000 & 0.5418 & 0.1272 & NS & 0.4359 & 0.4000 \\
\hline & R: ACATCCTGTCGTGCCTTGTT & & & & & & & & & & \\
\hline 286896 & F: TTTTGAGAAACCTTTTGCGAA & 275 & 59 & $\mathrm{~T} / \mathrm{C}$ & 92 & 0.1000 & 0.2291 & 0.3782 & NS & 0.2138 & 0.0750 \\
\hline & R: TCGGAAGGTGAGGTTAAACGT & & & & & & & & & & \\
\hline 286910 & F: TTCCATTTGTCAAATTCATCGAT & 352 & 60 & $\mathrm{C} / \mathrm{T}$ & 27 & 0.1000 & 0.1873 & 0.2897 & NS & 0.1769 & 0.0500 \\
\hline & R: ACACTGTTGCCCAAAATGATGG & & & & & & & & & & \\
\hline 286921 & F: CCCAGTACCCGTGTCATCAG & 268 & 60 & $\mathrm{~T} / \mathrm{C}$ & 97 & 0.1000 & 0.1873 & 0.2897 & NS & 0.1769 & 0.0500 \\
\hline & R: GCCATTGCACTGATGACGAC & & & & & & & & & & \\
\hline 286938 & F: TCAGTTTGAATCACTGTGACA & 299 & 60 & $\mathrm{G} / \mathrm{A}$ & 86 & 0.1250 & 0.2066 & 0.2332 & NS & 0.1910 & 0.0875 \\
\hline & R: GTCAGTTTTCTGCCACAGCG & & & & & & & & & & \\
\hline 286939 & F: ATTCCCTAAGCCCTAGTGCC & 288 & 60 & $\mathrm{C} / \mathrm{T}$ & 53 & 0.2250 & 0.5282 & 0.3841 & NS & 0.4430 & 0.3125 \\
\hline & R: GGATCACGATAAAACGCGGC & & & & & & & & & & \\
\hline 286945 & F: АССАTTTTCTCCACGTCCCT & 321 & 60 & $\mathrm{G} / \mathrm{T}$ & 91 & 0.1750 & 0.4839 & 0.4562 & NS & 0.4298 & 0.1375 \\
\hline & R: CGTTCTGCCAGGAGGTGATT & & & & & & & & & & \\
\hline 286946 & F: TCGGACAATAGTTAGAGCAGCT & 255 & 60 & $A / G$ & 97 & 0.1250 & 0.1649 & 0.1240 & NS & 0.1553 & 0.0625 \\
\hline & R: AAACCAGCAGGATCCATGCA & & & & & & & & & & \\
\hline 286949 & F: GTTCGAAAACCAGCAGAATCCA & 256 & 60 & $\mathrm{~T} / \mathrm{C}$ & 95 & 0.1000 & 0.2266 & 0.3750 & NS & 0.2075 & 0.1000 \\
\hline & & & & Page & & & & & & & \\
\hline
\end{tabular}


R: AAACCAGCAGCCGATCAGAA

286953 F: GCAACAGGGTCTTACTCGCT

R: CCGCGTTGTTTCAGTCGATC

286957 F: ATTCATGCCCTTTGGACCCC

R: TGACGGCAGGCCAGTTTTTA

286963 F: CTTCGCCAGCTCTACACTCC

R: GAGACGTGTGGAAGATGGGG

286970 F: TTCAGTTGTCGGGGAACTCG

$\begin{array}{lll}303 & 60 & \mathrm{~T} / \mathrm{G} \quad 86\end{array}$

$\begin{array}{llll}0.3000 & 0.6127 & 0.3154 & \text { NS }\end{array}$

$0.5270 \quad 0.3500$

R: ACACTTTCGTTTCTGAAGCGT

286981 F: CGGTCAGCAATTATGGAAATGCA

$274 \quad 60 \quad \mathrm{~A} / \mathrm{C} \quad 55$

$\begin{array}{lll}0.3250 & 0.4617 & 0.1518 \quad N S\end{array}$

$0.3881 \quad 0.2625$

R: CAGACGACATGCTCTCGTGT

286985 F: TGGGGTTTTACGACGTACTCA

$30460 \quad \mathrm{C} / \mathrm{T} \quad 79$

$\begin{array}{llllll}0.0750 & 0.3256 & 0.6156 & \text { NS } & 0.2973 & 0.1125\end{array}$

R: TGCTGGATGTTGCTGTTTTCC

286986 F: TTCGCTGATCTGGGTGCTAC

$337 \quad 61 \quad \mathrm{~A} / \mathrm{C} \quad 98$

$\begin{array}{llll}0.3250 & 0.4560 & 0.1387 & \text { NS }\end{array}$

$0.4068 \quad 0.1625$

R: AAGAAGAAACGGCGCTGTCA

286993 F: GCAATTGTTGATTCCAGCGC

28360 G/A 110

$\begin{array}{lll}0.1500 & 0.3430 & 0.3798\end{array}$ NS

$0.3114 \quad 0.0750$

R: TGAGAGTTTGGCGCTGGAAT

286997

F: TCGTTTTTCCATAATACATTCATTCGA

$\begin{array}{llll}335 & 60 & \text { G/A } & 34\end{array}$

$\begin{array}{llll}0.1000 & 0.2266 & 0.3750 & \text { NS }\end{array}$

$0.2075 \quad 0.1000$

R: TGATTGAGGTTTTCCGCCGT

287004 F: ACAATAACACGCTTTAACACACA

$278 \quad 60 \quad \mathrm{~A} / \mathrm{T} \quad 82$

$\begin{array}{llll}0.0750 & 0.1655 & 0.3590 & \text { NS }\end{array}$

$0.1569 \quad 0.0375$

R: CGATCGCATCGCCAACAATT

287008 F: CGACACTTGTTAACGTTCGTCC

$\begin{array}{llll}329 & 60 & \mathrm{C} / \mathrm{T} & 40\end{array}$

$\begin{array}{lll}0.1500 & 0.2684 & 0.2670 \quad \text { NS }\end{array}$

$0.2469 \quad 0.1000$

R: GGTGGAGAAACCCCTGGAAG

287013 F: TCATTGCATTAGCCAACAGC

$29659 \quad$ T/C 55

$\begin{array}{llll}0.1000 & 0.3430 & 0.5375 & \text { NS }\end{array}$

$0.3114 \quad 0.1250$

R: AGCTTCCTGATGTTGCTGTTG

\begin{tabular}{|c|c|c|c|c|c|c|c|c|c|c|c|}
\hline \multirow[t]{2}{*}{287019} & F: ACGATTAGAGTGGGACATGGA & \multirow[t]{2}{*}{292} & \multirow[t]{2}{*}{60} & \multirow[t]{2}{*}{$\mathrm{T} / \mathrm{A}$} & \multirow[t]{2}{*}{74} & \multirow[t]{2}{*}{0.0750} & \multirow[t]{2}{*}{0.2079} & \multirow[t]{2}{*}{0.4553} & \multirow[t]{2}{*}{ NS } & \multirow[t]{2}{*}{0.1942} & \multirow[t]{2}{*}{0.0375} \\
\hline & R: GCTCTGCCACGCTTGTTTAG & & & & & & & & & & \\
\hline \multirow[t]{2}{*}{287020} & F: TTAACCACCAGCTGCGACAA & 328 & 60 & $\mathrm{G} / \mathrm{A}$ & 69 & 0.1250 & 0.2491 & 0.3171 & NS & 0.2308 & 0.0875 \\
\hline & R: GCTTCGCTTGTCCTCCTCTT & & & & & & & & & & \\
\hline \multirow[t]{2}{*}{287033} & F: TGGTTTCTCCTTCCATAAGTGT & 282 & 60 & $\mathrm{~T} / \mathrm{C}$ & 40 & 0.3250 & 0.3668 & 0.0541 & NS & 0.2966 & 0.2375 \\
\hline & R: AGCTCTTGTGAGGGATGTGC & & & & & & & & & & \\
\hline \multirow[t]{2}{*}{287041} & F: ACTTCAGCTTCACCACCCAG & 298 & 60 & $A / G$ & 90 & 0.1500 & 0.4101 & 0.4499 & NS & 0.3680 & 0.1500 \\
\hline & R: CGTGTGTGATGGACCGGTAA & & & & & & & & & & \\
\hline \multirow[t]{2}{*}{287045} & F: AGTCAAACAGCAACAACGGC & 262 & 60 & $\mathrm{~T} / \mathrm{C}$ & 66 & 0.2000 & 0.6367 & 0.5050 & * & 0.5501 & 0.4000 \\
\hline & R: CCGAAGCCTGTAATCCAGACA & & & & & & & & & & \\
\hline \multirow[t]{2}{*}{287057} & F: ACGAGTTACTATTTTATATGTTGAACG & 250 & 60 & $A / G$ & 110 & 0.2000 & 0.3759 & 0.2862 & NS & 0.3372 & 0.1500 \\
\hline & R: ACACCACAGCGTGCAATAGA & & & & & & & & & & \\
\hline \multirow[t]{2}{*}{287062} & F: ACAGCATATCATGACAAAGGTGT & 334 & 59 & $\mathrm{C} / \mathrm{G}$ & 99 & 0.0500 & 0.1873 & 0.5516 & NS & 0.1769 & 0.0500 \\
\hline & R: GCGTTTGCATTGAGTACGGT & & & & & & & & & & \\
\hline \multirow[t]{2}{*}{287065} & F: ACATTGTCATTTGCACCACA & 285 & 60 & $\mathrm{~A} / \mathrm{C}$ & 96 & 0.3500 & 0.4772 & 0.1383 & NS & 0.3813 & 0.3250 \\
\hline & R: TCTGAAGAACAAGGGTGCCC & & & & & & & & & & \\
\hline \multirow[t]{2}{*}{287083} & F: TTCGCCGAAATACAGCAAAT & 353 & 60 & $\mathrm{C} / \mathrm{G}$ & 49 & 0.0000 & 0.4101 & 0.9966 & NS & 0.3680 & 0.1500 \\
\hline & R: TTCGGCTGAAACTTGACGGA & & & & & & & & & & \\
\hline
\end{tabular}




\begin{tabular}{|c|c|c|c|c|c|c|c|c|c|c|c|}
\hline \multirow[t]{2}{*}{287083} & F: TCGCCGAAATACAGCAAATT & \multirow[t]{2}{*}{328} & \multirow[t]{2}{*}{59} & \multirow[t]{2}{*}{$\mathrm{T} / \mathrm{C}$} & \multirow[t]{2}{*}{106} & \multirow[t]{2}{*}{0.1750} & \multirow[t]{2}{*}{0.4782} & \multirow[t]{2}{*}{0.4479} & \multirow[t]{2}{*}{ NS } & \multirow[t]{2}{*}{0.4191} & \multirow[t]{2}{*}{0.2125} \\
\hline & R: TCTCGATTGTGCAAGCATGG & & & & & & & & & & \\
\hline \multirow[t]{2}{*}{287138} & F: GGGGCCCATAGCGTGAATT & 307 & 60 & $\mathrm{~T} / \mathrm{A}$ & 79 & 0.3750 & 0.5453 & 0.1626 & NS & 0.4378 & 0.4125 \\
\hline & R: TATAAATCCGCCAGTCCGCC & & & & & & & & & & \\
\hline \multirow[t]{2}{*}{287182} & F: TGAAACGTCTACTTTATAACCTGCT & 337 & 60 & $\mathrm{G} / \mathrm{C}$ & 64 & 0.1000 & 0.4266 & 0.6118 & NS & 0.3651 & 0.2250 \\
\hline & R: GTTGTCCACCTAGCCACGAA & & & & & & & & & & \\
\hline \multirow[t]{2}{*}{287192} & F: TTCCCACCAACTAGCGTCTG & 313 & 60 & $\mathrm{~A} / \mathrm{T}$ & 107 & 0.0500 & 0.1873 & 0.5516 & NS & 0.1769 & 0.0500 \\
\hline & R: TGAGGAAAGTTTGGACCATTTGA & & & & & & & & & & \\
\hline \multirow[t]{2}{*}{287224} & F: TCCAGGCAATATAATAAGTGGCGA & 311 & 60 & $\mathrm{C} / \mathrm{T}$ & 94 & 0.0750 & 0.1206 & 0.2179 & NS & 0.1155 & 0.0375 \\
\hline & R: TATACAACAGTGCCGCGTGT & & & & & & & & & & \\
\hline \multirow[t]{2}{*}{287240} & F: TCTGTCCTCGAAGACCACCA & 273 & 60 & $\mathrm{C} / \mathrm{A}$ & 47 & 0.0500 & 0.0978 & 0.2936 & NS & 0.0949 & 0.0125 \\
\hline & R: TGCCATATATTCTTGCCCCGG & & & & & & & & & & \\
\hline \multirow[t]{2}{*}{287283} & F: TGCAAGGCATAACATAAAAGAAA & 259 & 59 & $\mathrm{G} / \mathrm{A}$ & 71 & 0.1000 & 0.1861 & 0.2875 & NS & 0.1736 & 0.0750 \\
\hline & R: TCTGGCGTTGGTTTCTGAGA & & & & & & & & & & \\
\hline \multirow[t]{2}{*}{287292} & F: AGGTCATAAATATAACGGATGACT & 310 & 59 & $\mathrm{G} / \mathrm{A}$ & 69 & 0.0750 & 0.0731 & -0.0102 & NS & 0.0696 & 0.0375 \\
\hline & R: ACTTCGAGTTTAGGTGCCTGA & & & & & & & & & & \\
\hline \multirow[t]{2}{*}{287298} & F: TCCCCAATCGTCAAAATGCA & 355 & 60 & $\mathrm{G} / \mathrm{T}$ & 45 & 0.2500 & 0.4595 & 0.2735 & NS & 0.3981 & 0.2250 \\
\hline & R: ACATTCGGTATTCCAGAACCTGT & & & & & & & & & & \\
\hline \multirow[t]{2}{*}{287301} & F: АСАССТССАТTTTACAАACTGTCA & 348 & 60 & $\mathrm{~A} / \mathrm{T}$ & 106 & 0.0750 & 0.2079 & 0.4553 & NS & 0.1942 & 0.0375 \\
\hline & R: GCGGCGTTAAACCCCAATC & & & & & & & & & & \\
\hline \multirow[t]{2}{*}{287340} & F: TCCAACTGTTACAGATATACATCAACT & 282 & 59 & $\mathrm{C} / \mathrm{A}$ & 50 & 0.2250 & 0.4136 & 0.2775 & NS & 0.3564 & 0.2125 \\
\hline & R: AGAACCCACTGAAATTTGTTGCT & & & & & & & & & & \\
\hline 287355 & F: AGCATTTGCAAAGTGGAGTGC & 330 & 60 & $\mathrm{C} / \mathrm{G}$ & 86 & 0.1500 & 0.4899 & 0.5176 & NS & 0.4275 & 0.2250 \\
\hline & R: GCTGGCAGATCTAGCTGTCC & & & & & & & & & & \\
\hline 287387 & F: TTGTGCAGTTTTTAGAAAATGGA & 314 & 56 & $\mathrm{~T} / \mathrm{C}$ & 90 & 0.1500 & 0.4342 & 0.4726 & NS & 0.3803 & 0.2000 \\
\hline & R: ACACATTTCTTAACGTTGGGT & & & & & & & & & & \\
\hline 287412 & F: TCCAAAGACAGCCTTAAGTGACT & 278 & 60 & $\mathrm{G} / \mathrm{A}$ & 81 & 0.1000 & 0.1430 & 0.1640 & NS & 0.1359 & 0.0500 \\
\hline & R: ACAACTGAACCTGCCCGTAC & & & & & & & & & & \\
\hline 287413 & F: ATCCACCGTTCGAAAGCAGT & 310 & 60 & $\mathrm{~A} / \mathrm{C}$ & 108 & 0.2000 & 0.3392 & 0.2403 & NS & 0.3029 & 0.1500 \\
\hline & R: TAATTCAATCCGCCTGGGCT & & & & & & & & & & \\
\hline 287424 & F: TCGAGCAAAAATTAATTGAAAGCT & 283 & 60 & $\mathrm{~A} / \mathrm{G}$ & 109 & 0.2250 & 0.4250 & 0.2863 & NS & 0.3796 & 0.1625 \\
\hline & R: GTGTTAAGGCTGGGGAGGAC & & & & & & & & & & \\
\hline 287438 & F: TCCTTGAAAGTTTCACGGGA & 321 & 60 & $\mathrm{C} / \mathrm{A}$ & 91 & 0.0500 & 0.1434 & 0.4509 & NS & 0.1367 & 0.0125 \\
\hline & R: GGCACGAGATGTCCAAGGAA & & & & & & & & & & \\
\hline 287440 & F: TTCTTTCTGTAAACATATGCCCA & 314 & 57 & $\mathrm{~T} / \mathrm{C}$ & 71 & 0.1750 & 0.3554 & 0.3243 & NS & 0.3150 & 0.1625 \\
\hline & R: ACCATAACAAAAACAGAAGCCA & & & & & & & & & & \\
\hline 287443 & F: ACTGATCAGCGTAACTACAGGT & 353 & 60 & $\mathrm{~A} / \mathrm{G}$ & 97 & 0.2000 & 0.5025 & 0.4137 & NS & 0.4267 & 0.2750 \\
\hline & R: AGCTTGGCCAGAAACATCCA & & & & & & & & & & \\
\hline 287446 & F: ATTCACTGTTTACTGTTTTTGTTTT & 284 & 60 & $\mathrm{G} / \mathrm{T}$ & 84 & 0.1000 & 0.1873 & 0.2897 & NS & 0.1769 & 0.0500 \\
\hline & R: GATTGTGTCATCACGCGGTC & & & & & & & & & & \\
\hline 287478 & F: GGTGTTGGTTTCATGCGACG & 329 & 60 & $A / G$ & 104 & 0.1250 & 0.5953 & 0.6454 & $\star \star \star ~$ & 0.5226 & 0.2125 \\
\hline & R: ACGTCACACAAAGGGGCATA & & & & & & & & & & \\
\hline 287512 & F: TTCCTGACCCTGCCAACATC & 345 & 60 & $\mathrm{G} / \mathrm{T}$ & 27 & 0.0250 & 0.2079 & 0.7469 & NS & 0.1942 & 0.0375 \\
\hline & & & & Page & & & & & & & \\
\hline
\end{tabular}


R: ACACCAGTCAAAAGATCTTCTGA

\begin{tabular}{|c|c|c|c|c|c|c|c|c|c|c|c|}
\hline \multirow[t]{2}{*}{287513} & F: ACAGTAAGATTAAGTTTACCTGAGCT & \multirow[t]{2}{*}{336} & \multirow[t]{2}{*}{60} & \multirow[t]{2}{*}{$\mathrm{G} / \mathrm{A}$} & \multirow[t]{2}{*}{48} & \multirow[t]{2}{*}{0.0750} & \multirow[t]{2}{*}{0.1206} & \multirow[t]{2}{*}{0.2197} & \multirow[t]{2}{*}{ NS } & \multirow[t]{2}{*}{0.1155} & \multirow[t]{2}{*}{0.0375} \\
\hline & R: GGCCCATAATGGAGCTCGAA & & & & & & & & & & \\
\hline \multirow[t]{2}{*}{287514} & F: TTGCTGGTCATCTGAGGTGT & 341 & 59 & $\mathrm{C} / \mathrm{T}$ & 51 & 0.1000 & 0.1405 & 0.1617 & NS & 0.1291 & 0.0750 \\
\hline & R: ACAAGTGACAGTGACCATCA & & & & & & & & & & \\
\hline \multirow[t]{2}{*}{287523} & F: GGTTTAAACCCATGCGCGTT & 281 & 60 & $\mathrm{~T} / \mathrm{C}$ & 53 & 0.3500 & 0.5051 & 0.1662 & NS & 0.3969 & 0.3750 \\
\hline & R: GCTGACGGTGTTGTTGTTGG & & & & & & & & & & \\
\hline \multirow[t]{2}{*}{287534} & F: AGAGTGAAGGGACTCAACCA & 304 & 60 & $T / G$ & 110 & 0.0750 & 0.2497 & 0.5247 & NS & 0.2323 & 0.0625 \\
\hline & R: TGCGCATCCATGGCTTATCT & & & & & & & & & & \\
\hline \multirow[t]{2}{*}{287536} & F: АССTTATCTTTTAAATGCACCTGT & 259 & 60 & $\mathrm{G} / \mathrm{A}$ & 100 & 0.2000 & 0.4658 & 0.3805 & NS & 0.4120 & 0.2000 \\
\hline & R: TCGTTCATGCAGACAAGAGATGA & & & & & & & & & & \\
\hline \multirow[t]{2}{*}{287578} & F: TGTGCGCCCTGAATTACAGT & 331 & 60 & $\mathrm{~T} / \mathrm{G}$ & 54 & 0.1000 & 0.0962 & -0.0164 & NS & 0.0905 & 0.0500 \\
\hline & R: CGTGGTGCACAGTCAATTCC & & & & & & & & & & \\
\hline \multirow[t]{2}{*}{287581} & F: TTCCAGTGGTGAATCCCGAA & 353 & 60 & $A / G$ & 55 & 0.1250 & 0.4206 & 0.5300 & NS & 0.3706 & 0.1875 \\
\hline & R: ATAGCACTTTCGCCTGGAGC & & & & & & & & & & \\
\hline
\end{tabular}

$T_{m}$ annealing temperature, $H_{O}$ observed heterozygosity, $H_{E}$ expected heterozygosity, $F_{I S}$ inbreeding coefficient, $H W E P$ results for Hardy-Weinberg Equilibrium text, $P I C$ polymorphism information content, MAF minor allele frequency, NS non-significant, ${ }^{\star} P<0.05,{ }^{\star \star} \mathrm{P}<0.01, \star \star \star P<0.001$ 